ISSN (print) 0867-2008 / ISSN (online) 2391-75 I2

DOI: http://dx.doi.org/10.12775/OM.2019.011

JUHAN KREEM*

Tallinna Linnaarhiiv

Tolli 6

E-10133 Tallinn

Estonia

Juhan.Kreem@tallinnlv.ee

DMitriy Weber**

Institut Philosophii

Sankt-Petersburskiy gosudarstvennyy universitet

St. Petersburg, Mendeleevskaya liniya, 5

199034

Russian Federation

d.veber@spbu.ru

\title{
EINE NEUE QUELLE ZUM AUFSTIEG GOTTHARD KETTLERS INS AMT DES LIVLÄNDISCHEN ORDENSMEISTERS
}

\section{KEYWORDS}

history; military orders; I $6^{\text {th }}$ century; Teutonic Order; Livonian War; Gotthard Kettler; administration; diplomacy

\begin{abstract}
A New Source on the Advance of Gotthard Kettler into the Office of the Master of the Teutonic Order in Livonia

Gotthard Kettler was elected coadjutor (co-ruler and successor) of the master of the Teutonic Order in Livonia in July I 558 . The Order in Livonia was in deep crisis because of the war with the Muscovy, and the old master Wilhelm von Fürstenberg was unable to manage it. In these circumstances Kettler strived with all methods for the office of the master. The chronicles of the period date the final change in the leadership of the Order as of September 1559. Earlier research has noted, however, that Kettler occasionally used the title of the master as early as in February 1559. The charter published here is another evidence on Kettlers aspirations. A group of
\end{abstract}

* ORCID: https://orcid.org/o0oo-000 I-8465-763X

** ORCID: https://orcid.org/o000-0002-6I 58 -770 I 
officials of the Teutonic Order in Livonia confirm herewith that they have accepted Gotthard Kettler as their new master on 22 February 1559. Legal ramifications of this charter are not straightforward. The main ratio behind it seems to be the support to Kettler for his mission in Poland, where he was searching for help against Muscovy, and needed to legitimise his negotiating position. Final change in the leadership of the Order still took more time, until the retirement conditions of the old master Fürstenberg were settled.

otthard Kettler, der letzte Landmeister des livländischen Zweigs des Deutschen Ordens und der erste Herzog von Kurland, ist eine umstrittene Person in der Geschichte der Baltischen Lande. Kettler wirkte während der ersten Jahre des Livländischen Krieges und wurde im Sommer I 558 zum Koadjutor des alten Meisters Wilhelm von Fürstenberg gewählt. Während seiner Koadjutorenschaft hat er schrittweise den alten Meister zurückgedrängt und im Spätherbst I 56 I das durch den Krieg dezimierte Ordensterritorium Polen unterworfen. Mit diesem Akt wurde der Orden in Livland abgeschafft, Kettler selbst aber eignete sich aus dem ehemaligen Ordensbesitz das Herzogtum Kurland an. Unter diesen Umständen gehen die Meinungen darüber, ob Kettler nun als Verräter oder als Retter des Landes zu sehen ist, auseinander. Publikationen über Kettler bestehen zumeist aus kürzeren Darstellungen in Aufsatzformat, eine modernen Ansprüchen genügende Biografie steht aber noch aus. ${ }^{1}$

Die Übergabe des Meisteramtes von Fürstenberg an Kettler wird nach den Chroniken und sonstigen indirekten Quellen auf den September I 599 datiert. ${ }^{2}$ Im Folgenden soll ein Dokument vorgestellt werden, das mehr Licht auf die Umwälzungen im Orden im Frühjahr I 559 werfen soll. Es befindet sich gegenwärtig im

I Theodor Schiemann, Historische Darstellungen und Archivalische Studien. Beiträge zur baltischen Geschichte (Hamburg: Gebr. Behre, I 886), 91-1 02; Johann Seibertz, "Gotthard Kettler letzter Ordensmeister in Livland und erster Herzog von Kurland," Zeitschrift für vaterländische Geschichte und Altertumskunde Westfalen 29, no. 2 ( I 871): I-92; Friedrich von Klocke, "Gotthard Kettler," Westfälische Lebensbilder 2 (193 I): 4I I-438; Heinz Mattiesen, "Gotthard Kettler und die Entstehung des Herzogtums Kurlands," in Baltic History, hrsg. v. Mardi Valgemäe, William L. Winter und Arvids Ziedonis (Columbus, 1974), 49-59; Norbert Angermann, Gotthard Kettler (Bonn: Bund der Vertriebenen, 1987); Udo Arnold, "Hochmeister Albrecht von Brandenburg und Landmeister Gotthard Kettler. Ordensritter und Territorialherren am Scheideweg in Preußen und Livland," in The military orders and the Reformation. Choices, state buildings and the weight of tradition, hrsg. v. Johannes A. Mol, Klaus Militzer und Helen J. Nicholson (Hilversum: Verloren, 2006), i I-3 I. Über das Bild des Gotthard Kettler siehe. Sebastian Plüer, "Gotthard Kettler, letzter Ordensmeister in Livland und erster Herzog von Kurland - eine umstrittene Persönlichkeit in der Geschichtsschreibung," in Das Herzogtum Kurland I56I-I795. Verfassung, Wirtschaft, Gesellschaft, Bd. II, hrsg. v. Erwin Oberländer (Lüneburg: Nordostdeutsches Kulturwerk 1993), I I - 53.

2 Vor allem Johann Renner, Livländische Historien I556-I56I, hrsg. v. Peter Karstedt (Lübeck: Max Schmidt-Römhild, I 953), 66. 
Archiv des Instituts für Geschichte der Russischen Akademie der Wissenschaft in Sankt Petersburg (ASPIG). ${ }^{3}$ Es gehört zu einer Quellensammlung mit dem Titel „Materialien zur Geschichte Livlands“, die Urkunden zur Geschichte dieser Region aus dem Mittelalter und aus der Frühneuzeit beinhaltet. ${ }^{4}$ Die Maße des Folianten weisen eine Höhe von $36,6 \mathrm{~cm}$ und eine Breite von $22 \mathrm{~cm}$ auf. Er enthält 288 nummerierte Blätter aus Papier und Pergament und gehörte seit I 838 der Archäographischen Kommission. 5 Diese Quellensammlung wurde vom Vizegouverneur von Grodno im Archiv des beschlagnahmten Landguts Deretschin des Fürstens Sapieha gefunden. ${ }^{6}$ Früher befand sich dieses Archiv auf einem anderen von Sapiehas Landgütern, in Rozan (pol. Różan). ${ }^{7}$ Das Dokument, das uns interessiert, gehört zur Abteilung der Sammlung, die der Geschichte des Deutschen Ordens in Livland gewidmet ist. Es heißt: Przymerza y Inne Akta Publiczne Krzyżaków Inflantskich / Bündnisse und weitere öffentliche Akten des Deutschen Ordens in Livland. ${ }^{8}$

Das in einem Konvolut eingebundene Pergamentdokument ist $30 \mathrm{~cm}$ hoch und $54 \mathrm{~cm}$ breit. In der Plica $(6 \mathrm{~cm}$ hoch $)$ sind Einschnitte für sechs Siegel, die Sie-

3 Sankt Petersburg, Archiv des Instituts für Geschichte der Russischen Akademie der Wissenschaft in Sankt Petersburg [im folgenden: ASPIG RAW], Westeuropäische Abteilung, Sammlung 33, Nr. 483, fol. 79 r.

4 Über dieses Konvolut siehe Dmitriy Weber, "Quellen zur Geschichte des Deutschen Ordens aus St. Petersburger Sammlungen," in Editionswissenschaftliches Kolloquium 2017. Quelleneditionen zur Geschichte des Deutschen Ordens und anderer geistlicher Institutionen, hrsg. v. Helmut Flachenecker, Janusz Tandecki und Krzysztof Kopiński (Toruń: Wydawnictwo Naukowe Uniwersytetu Mikołaja Kopernika, 20 I7), 97-ı 08; Alexander Rogatschewski, "Baltische Rechtsdenkmäler des 13. bis I 8. Jahrhunderts im Archiv des St. Petersburger Instituts für Geschichte der Russischen Akademie der Wissenschaften," in Einheit und Vielfalt in der Rechtsgeschichte im Ostseeraum, hrsg. v. Marju Luts-Sootak, Sanita Osipova und Frank L. Schäfer (Frankfurt am Main: Peter Lang, 20 I2), 20 I-2 I 7, hier 207-2 I I; ders., "Baltische Staats- und Rechtsgeschichte des I 3 . bis I 8. Jahrhunderts in den Handschriftensammlungen von St. Petersburg," Blätter für Deutsche Landesgeschichte I 45/ I 46 (2009/20 I 0): 2 I 7-280, hier 252-256.

5 ASPIG RAW, Westeuropäische Abteilung, Sammlung 33, Nr. 483, fol. 229 r.

6 Sankt Petersburg, Sankt Petersburger Außenstelle des Archivs der Russischen Akademie der Wissenschaften, Bestand I 33 , Inv. I, Nr. 36, fol. 7r-I Ir. Dazu siehe auch Marjan Morelowski, "Zbiory dereczyńskie Sapiehów," in Sprawozdania z Czynności i Posiedzeń PAU 29, Nr. 10 (1924): 6-9.

7 Franciszek Radziszewski, Wiadomość historyczno-statystyczna o znakomitszych bibliotekach $i$ archiwach publicznych i prywatnych (Kraków: Drukarnia Wł. L. Anczyca I 875), I0, 72; Maciej Matwijów "Rękopiśmienne zbiory materiałów życia publicznego XVII-XVIII w. w bibliotekach i archiwach wileńskich," in Z Badań nad Ksiązka i Ksiegozbiorami Historycznymi, hrsg. v. Jacek Puchalski, Agnieszka Chamera-Nowak und Dorota Pietrzkiewicz (Warszawa: Wydział Dziennikarstwa, Informacji i Bibliologii Uniwersytetu Warszawskiego, 20 1 7), 95- 1 08, hier 96.

8 ASPIG RAW, Westeuropäische Abteilung, Sammlung 33, Nr. 483, fol. 65 r- I 16 v. 
gel sowie das Band fehlen. Auf der Plica sind die eigenhändigen Unterschriften der Gebietiger: Philipp Schall van Bell,, Lantmarschalck D. O. tho Lifflandt, Bernt van Smerthen ${ }^{10}$ egen hanth, Hynk Stadynk, ${ }^{11}$ Hynrych van Galenn, ${ }^{12}$ vaghet thom Boyßenborch D. O., Cristof Siberg, ${ }^{13}$ Claus von der Strithorst ${ }^{14}$. Auf dem Versoseite ist ein Vermerk mit der frühneuzeitlichen handschriftlichen Notiz: Consensus ordinis in cessionem administris Firstenbergii Kettlero I559 die 22 Februarii. Ordo cruciferorum consentit ut Vilhelmus Firsztenbergius magister ordinis, officium suum resignet in personam Gothardt Ketler 1559.

In dieser Urkunde wird Kettler somit am 22. Februar I 559 von den Unterzeichnenden und Siegelnden neben dem alten Meister Wilhelm von Fürstenberg als regierender Meister des Ordens in Livland bestätigt. Das Markanteste ist, dass die Urkunde auf ein halbes Jahr vor der bisher bekannten Übergabe des Amtes auf dem Ordenskapitel in Wenden am 17. September datiert wird. ${ }^{\text {Is }}$

Davon, dass Kettler sich schon im Frühjahr I 559 Meister nannte, zeugen auch einige Güterurkunden. Eine der Urkunden vom Io. Februar 1559 wird im Lettischen historischen Staatsarchiv aufbewahrt, ${ }^{16}$ die andere, vom i 8. Februar I 559, wurde veröffentlicht. ${ }^{17}$ In beiden Dokumenten bezeichnet sich Gotthard Kettler schon als: Von Gottes gnadenn wir Godthardt, meister des ritterlichen Teutschen ordenns $z w$ Liefflanndt [...]. Der zweite Brief ist für uns sowohl we-

9 Philipp Schall von Bell, Landmarschall des Deutschen Ordens in Livland I 558- I 560 , geriet in russische Gefangenschaft und wurde in Moskau hingerichtet, vgl. Ritterbrüder im livländischen Zweig des Deutschen Ordens, hrsg. v. Lutz Fenske und Klaus Militzer (Köln: Böhlau, I 993), Nr. 760.

10 Bernhard von Schmerten, Vogt von Jerwen I 55 I-1 562, vgl. Ritterbrüder, hrsg. v. Fenske und Militzer, Nr. 8 I 8.

I Heinrich Steding, Komtur von Goldingen I 556-1 560, vgl. Ritterbrüder, hrsg. v. Fenske und Militzer, Nr. 834.

12 Heinrich von Galen, Vogt von Bauska 1558-1559, Komtur von Rujen i 559-1 560, geriet in russische Gefangenschaft und wurde in Moskau hingerichtet, vgl. Ritterbrüder, hrsg. v. Fenske und Militzer, Nr. 283.

13 Cristoph Syburg zum Busch, Vogt von Kandau I 557-1 560, gefallen in der Schlacht bei Ermes (Ërǵeme, Lettland), vgl. Ritterbrüder, hrsg. v. Fenske und Militzer, Nr. 869.

14 Klaus von Streithorst, Vogt von Grobin I 55 I - I 560 . Später Vasall in Kurland, vgl. Ritterbrüder, hrsg. v. Fenske und Militzer, Nr. 848.

is Vgl. Renner, Livlandische Historien, 66; Juhan Kreem, "Die inneren Voraussetzungen der Säkularisation des Deutschen Ordens in Livland," Ordines Militares Colloquia Torunensia Historica. Yearbook for the Study of the Military Orders 22 (2017): 93-108, hier 98.

16 Rìga, Latvijas Valsts vēstures arhīvs, F. 556 I, Best. 4, Nr. 4 I 8.

17 "Aus der Brieflade von Zohden," Sitzungsberichte der Kurländischen Gesellschaft für Literatur und Kunst (Mitau: J. F. Steffenhagen u. Sohn, I 888), Anhang, S. 3-5. Vgl. auch Kurländische Güterurkunden, zugegriffen am I 4. Juli 20 I 7, https://www.herder-institut.de/bestaende-digitale-angebote/datenbanken/kurlaendische-gueterurkunden/urkundentext.html?nr= I 4 I 6. 
gen des Namens des neuen Lehnsmannes selbst als auch wegen seines Datums interessant. Bei diesem handelt es sich um Christian Schröder, der zu diesem Zeitpunkt der Sekretär von Heinrich von Galen, dem Vogt von Bauske (Bauska, Lettland), war, dessen Unterschrift auch auf der uns hier beschäftigenden Bestätigungsurkunde Kettlers steht.

Zwei weitere Beispiele dafür, dass sich Kettler im Frühjahr I 599 Meister nannte, sind aus dem Bereich der auswärtigen Politik bekannt. Das erste ist ein Memorial für Gotthard Kettler für seine Gesandtschaft in Polen, wo er um Hilfe werben wollte, und als [...] Maister des Ritterlichenn Teutschenn Ordenns zu Lifflanndtt [...] tituliert ist. ${ }^{18}$ Diese Urkunde stammt vom 2 r. Februar aus Riga, d. h., sie wurde einen Tag früher als die hier veröffentlichte Urkunde unterzeichnet. Das zweite Beispiel ist ein Brief vom I 2. März von Kettler an die Gesandten in Schweden Rembert Gilsheim und Salomon Henning. ${ }^{19}$ Eine weitere Gruppe von Urkunden stellen die Briefe dar, die nicht von Kettler, aber von anderen Personen geschrieben worden sind. Dazu gehören z. B. ein Brief von Thomas Horner an Herzog Albrecht, in dem Kettler nicht nur als Koadjutor, sondern auch als erwählter Meister tituliert ist, ${ }^{20}$ oder ein Bericht von Gesandten des Erzstifts Riga aus Insterburg an Herzog Albrecht über die Verhandlungen, in denen er der neue Meister genannt wird. ${ }^{21}$

Die hier veröffentlichte Urkunde stellt einen weiteren Beleg für die Ambitionen Kettlers dar, schon im Frühjahr i 559 als regierender Meister zu fungieren. Ihr historischer und rechtlicher Inhalt muss jedenfalls erläutert werden. Erstens bilden die beteiligten Personen, der Landmarschall Philipp Schall von Bell, der Vogt von Jerwen (Järvamaa, Estland), Bernard von Schmerten, der Komtur von Goldingen (Kuldīga, Lettland), Heinrich Steding, der Vogt von Bauske, Heinrich von Galen, der Vogt von Kandau (Kandava, Lettland), Cristoph Syburg zu Wischlingen und der Vogt von Grobin (Grobin,a, Lettland), Klaus Streithorst, nicht den ganzen Gebietigerrat. Auch wenn ein beträchtlicher Teil der Nord-Estnischen Ordensterritorien (Narva, Neuschloss (Vasknarva, Estland), Wesenberg (Rakvere, Estland) und Tolsburg (Toolse, Estland) zu dieser Zeit schon verloren war, waren die Gebietiger in Marienburg (Alūksne, Lettland), Pernau (Pärnu, Estland), Soneburg (Maasilinn, Estland), Doblen (Dobele, Lettland), Windau (Ventspils,

18 Quellen zur Geschichte des Untergangs livländischer Selbständigkeit, Bd. 3, hrsg. v. Carl Schirren (Reval: Franz Kluge, i 863), Nr. 334.

19 Quellen, hrsg. v. Schirren, Nr. 34I.

20 Herzog Albrecht und Livland (I55I-I557). Regesten aus dem Herzogliches Briefarchiv und den Ostpreussischen Folianten, bearb. v. Stefan Hartmann, Veröffentlichungen aus den Archiven Preußischer Kulturbesitz 57 (Köln-Weimar-Wien: Böhlau, 2005), Nr. 2428.

${ }_{21}$ Herzog Albrecht und Livland, hrsg. v. Hartmann, Nr. 2473. 
Lettland), Selburg (Sēlpils, Lettland) und Rositten (Rezekne, Lettland) noch im Amt, blieben aber aus unbekannten Gründen hier fern. Unsere Urkunde wurde wohl von einer Kerngruppe der Gebietiger besiegelt, wichtige Stimmen aus dem Gebietigerrat, vor allem vom Komtur von Marienburg, fehlen aber. Wir wissen nicht wie artikuliert die Opposition zu diesem Beschluss war und ob dessen Legitimität in Frage gestellt worden ist, man bekommt allerdings den Eindruck, dass der Gebietigerrat nicht einig war.

Parteibildungen waren kein Novum im Orden; in diesen Jahren ging es vor allem um die Richtung, in welcher man für Livland Unterstützung finden sollte. Der alte Meister Wilhelm von Fürstenberg stand in keinem guten Verhältnis zu PolenLitauen. Die Wurzeln für Fürstenbergs Ressentiment mögen in seiner früheren Funktion als Komtur von Dünaburg (Daugavpils, Lettland) an der Grenze liegen. In der Koadjutorfehde im Jahre I 556 eskalierten die Diskrepanzen zwischen dem Landmeister des Ordens und dem Erzbischof von Riga, unterstützt von seinem Bruder Albrecht von Preußen und dem polnischen König, zu kriegerischen Konfrontationen mit Polen-Litauen. ${ }^{22}$ Fürstenberg suchte zum Zeitpunkt des Beginns des Livländischen Krieges nicht nur im Heiligen Römischen Reich, sondern auch in Dänemark nach Unterstützung. Es gab im Orden aber auch eine Gruppe, die eine Annäherung an Polen befürwortete, welcher Fürstenberg im Wege stand. ${ }^{23}$

22 Thomas Lange, Zwischen Reformation und Untergang Alt-Livlands. Der Rigaer Erzbischof Wilhelm von Brandenburg im Beziehungsgeflecht der livländischen Konföderation und ibrer Nachbarländer (Hamburg: Dr. Kovač, 20 I4), I 80-243; Jerzy Olewnik, "Polsko-pruski plan inkorporacji Inflant do monarchii jagiellońskiej z lat I $552-1555$ i jego pierwsze stadium realizacji," Komunikaty Mazursko-Warmińskie 4/ 46 (1979): 393-408; Stefan Hartmann, "Neue Quellen zur livländischen Koadjutorfehde I 55/6," in Aus der Geschichte Alt-Livlands, hrsg. v. Bernhart Jähnig und Klaus Militzer (Münster: LIT, 2004), 275-307; Wadim Popow, Alexander Filjuschkin, "Wojna koadjutorow i Poswolskije soglaschenija i 557," Studia Slavica et Balcanica Petropolitana I (2009): I 5 I - I 85 ; Juhan Kreem, "Netzwerke um Jasper von Münster. Der Deutsche Orden während der livländischen Koadjutorfehde," Ordines Militares Colloquia Torunensia Historica. Yearbook for the Study of the Military Orders I 9 (20 I 4): 73-86; Johannes A. Mol, "Traitor to Livonia? The Teutonic Orders' landmarshall Jasper von Munster and his action at the outset of the Livonian crisis, I 554-1 556," Ordines Militares Colloquia Torunensia Historica. Yearbook for the Study of the Military Orders I 9 (2014): 205-240; Madis Maasing, "Saare-Lääne ja koadjuutorivaenus: keskaegse Liivimaa viimased kodusõjad," Ajalooline Ajakiri 2 (2010): I I 5-I 52; Dmitriy Weber, "Pamphlet as a means of a propaganda in Baltic region in Early Modern time," Vestnik Sankt Perterburgsgovo Gosudarstvennovo Universiteta. Istoria 62/2 (2017), 29I-298.

23 In Polen hatte das Bild von Fürstenberg eine negative Konnotation. Es ist bemerkenswert, dass Koadjutor Wilhelm von Fürstenberg im katholischen Polen als der Anführer der Protestanten galt, die den katholischen Erzbischof Wilhelm stürzen wollten. Vor diesem religiösen Hintergrund wurde Fürstenberg von polnischem Chronist Martin Belsky angeklagt. Siehe z. B. Marcin Bielski, Kronika Polska Marcina Bielskiego (Sanok: Nakład i drukarnia Karola Pol- 
Unser Dokument berichtet, neben der Bestätigung Kettlers als Meister (allerdings noch neben Fürstenberg), von künftigen Verhandlungen mit dem König von Polen. Gerade in der Mission Kettlers nach Polen im Frühling I 559 ist der Entstehungskontext und die Bedeutung der Urkunde zu suchen. ${ }^{24}$ Kettler bekommt hier eine Blankovollmacht für ein Abkommen mit Polen, gleichzeitig erhielt seine Verhandlungsposition mit der Ernennung zum regierender Meister mehr Gewicht, da er im Namen des ganzen Livländischen Ordens sprechen und Zusagen machen konnte. Wenn so außenpolitisch die Position Kettlers eine verbriefte Bestätigung fand, waren die Machtverhältnisse innerhalb des Ordens in Livland deutlich komplizierter. Auch wenn Kettler nach seiner Rückkehr im September I 559 behauptete, dass seine Ernennung zum regierenden Meister Vorbedingung des schon geschlossenen Bündnisses mit Polen sei, verzögerte sich die eigentliche Machtübergabe weiter bis April I 560, bis die Versorgungsfrage des alten Meisters endlich geregelt worden war. ${ }^{25}$

Bei der folgenden Edition handelt es sich um eine wortgetreue Wiedergabe des Textes. Doch wurden für bessere Lesbarkeit einige editorische Eingriffe vorgenommen. Im Original unregelmäßige Interpunktion wurde normalisiert, Großbuchstaben wurden nur für die Satzanfänge und Eigennamen (Orts- und Personenamen, Nomina sacra, und Bezeichnungen für geistliche Orden) verwendet. Die Buchstaben $\mathrm{u} / \mathrm{v}$ und $\mathrm{i} / \mathrm{j}$ wurden nach ihrem Lautwert (Vokal oder Konsonant) wiedergegeben. Konsonantenhäufungen wurden beibehalten. Mit Ausnahme der Anredeformen (fürstliche Gnade und königliche Majestät) wurden Abkürzungen sowie allgemein gebräuchliche Siegel und Abbreviaturen (Deutsche Orden, et cetera) stillschweigend aufgelöst. Römische Zahlzeichen sind als arabische Zeichen wiedergegeben.

laka, I 856), i I 23. Ähnliche Aussagen werden von einem anderen polnischen Historiker des späten I 6. Jahrhunderts, Maciej Stryjkowski, gemacht. Vgl. Maciej Stryjkowski, Kronika Polska, Litewska. Zmodska i wszystkiej Rusi, Bd. 2 (Warszawa: Nakład Gustawa Leona Glücksberga, I 846), 409.

24 Siehe auch Lange, Zwischen Reformation und Untergang, 330-333.

25 Dazu mehr in Kreem, Die inneren Voraussetzungen. 


\section{QUELLENEDITION}

Riga, 22. Februar IS59.

Acht namentlich genannte Gebietiger des Deutschen Ordens in Livland bestätigen, dass die Gotthard Kettler an Stelle und neben den alten Meister Wilhelm von Fürstenberg zum Meister gewählt und bestätigt haben. Gleichzeitig geben die Gebietiger an Gotthard Kettler ein Vollmacht mit König von Polen um die Hilfe gegen die Moskoviten zu verhandeln.

Original: Archiv des Instituts für Geschichte der Russischen Akademie der Wissenschaft in Sankt Petersburg, Westeuropäische Abteilung, Sammlung 33, Nr. 483, fol. 79 r.

Größe/Umfang: $30 \mathrm{~cm}$ hoch $\times 54 \mathrm{~cm}$ breit; Stoff: Pergament; Siegel und Siegelbände nicht erhalten.

Wir, hiernachbenente Philipss Schall von Bell, landmarschalck zw Liefflant, Bernhart vonn Schmerten, vogt zw Jerven, Heinrich Stedting, commether zw Goldingen, Heinrich von Galen zuw Bawschenburg, Christoff Sieburgk zw Candow unnd Clauß vonn der Striethorst zw Gerbbin, vögte alle Teutzsches Ordennß, bekennen inn unnd mit diessen unseren offen versiegelten brieff vor uns, unnsers Ordenns verwanten unnd derselbigen nachkommen in Liefflandt jegen jedermennigelich, daß nach dem der hochwirdige grosmechtige furst unnd herr, herr Wilhelm Furstenbergk, meister desselbenn Ordenns zw Liefflandt, unser gnediger herr unnd oberster, unns genediglich zuerkennen geben, das ire furstliche g. vonn wegen der jtzbeschwerrlichen gelegenheit dieser armen provintz zw Liefflant, darein dieselb mit der behafften, unchristlichen, unbefugten Muskowitterschenn Reussischen emporungen ein zeitlang heer gerathen unnd leider nach diese stunde stecken, der obliegenden hochwichtigenn unnd vielfaltigen muhe, gescheffte unnd sorge halber, so auß solchen emporungen her flissen sein, auch daß ohne das ire f. g. mit einem zimblichen gutten betagten alter versehen unnd inn dem auch ringerung einer volnkomnen gesundheit vermercken theten, hinfurder gantz kommerlich unnd unmueglich der regierung obstenn, vergewalten unnd verwesen kontenn, unnd unß demnach umb erlassung derselben begirlich, genediger unnd bittlicher massen ahngesucht, wozu jegen wir auch obberurte erhebliche ursachenn erwogenn, irer f. g. begehrn stadt gegebenn unnd derselben tragendenn ambts unnd regierungk entlich erlassen habenn. Darauff auch wiederumb den hochwirdigen fursten und hern, herrn Godharten Kettlern, irer f. g. unlangst erweltenn coadiutorn unnd negsten successorn deß meisterthumbs, auch commethern zw Vellin unnd Revall, nhumals tzw einem bestindigenn regierenden herrn meister zw Liefflant einhelliger weise, neben hoch- 
gadachten unsern gnedigen herrn unnd obersten, denn alten, eligirt unnd aufgenhommen. Unnd aber hochgedachter etc. unser genediger herr, der altt, unnser Ritter Orde unnd wir, umb suchung einer wirklichen hulff, rhat unnd beistand unsere gesanten vor einer kurzen zeit an den durleuchtigsten grosmechtigen hochgebornen fursten und herrn, hern Sigismundum Augustum ${ }^{26}$ Konigen zw Poln unnd grosfursten zw Lyttawen etc., unsern genedigen herrn unnd nachbarn, abgefertigt, so dann unsers hoffens nhumehr bei irer Kon. M. ankommen sein mugen, so haben wir fur dißmal bei unns der sachenn erforderter gelegenheit nach zutreglicher unnd furderlicher zusein gerathen erachtet, daß nit allein zu einer hulff unnd beistands suchung, sondern auch zw erbawung, stifftung unnd ersetzung einer bestedigen zuuersicht, trost unnd freuntschafft hochgegedechter etc. furst, herr Godhart Kettler, itziger regierender herr meister unser gnediger herr unnd oberster, eigner persohn an hochstermelte kunigliche mat. zu Polen etc. sich begebe unnd verreise, wie wir ire f. g. dann hiemit auch abgefertigt habenn wollen. Demnach geben wir iren f. g. vor unns, unsern Ordenn und nachkommen inn Lieflant krafft diess briefs volnkommene gewalt unnd macht mit hochstgemelter kon. m. zu Polen etc. derselben reich Polen unnd grosfurstenthumb Lyttawen allerseitz unnd unwiederrufflich zuhandeln unnd zuschlissen, was zu rettung dieser armen provintz zu Lieffland itzigen bedruck nach immer gedeilich unnd nötig sein konne oder muge. Unnd was also hochgedachter unser gnediger herr meister vor unns unnd unseren Ritter Orden zw Liefflant ufnhemen unnd bewilligen werden, daß gereden neben irer $f$. g. wir vor unns unnd gemelten Orden stett, vest unnd unverbrochen zuhalten, ohne gefehrde. Zu urkund haben wir, obberurte landmarschalck, cumpthure und vögte, unsere gebreuchliche ambtsiegell ahn diessen brieff lassen hangen unnd mit eignen handenn unterschrieben. Geben zu Riga den 22. februari nach Christi geburtt der weniger tzall inn neun und funftzigsten Jhare.

\section{PRIMARY SOURCES:}

Sankt Petersburg. Archiv des Instituts für Geschichte der Russischen Akademie der Wissenschaft in Sankt Petersburg, Westeuropäische Abteilung, Sammlung 33, Nr. 483.

Rìga. Latvijas Valsts vēstures arhīvs, F. 556I, Best. 4, Nr. 4 I 8.

Sankt Petersburg. Sankt Petersburger Außenstelle des Archivs der Russischen Akademie der Wissenschaften, Bestand I 33 , Inv. I, Nr. 36.

"Aus der Brieflade von Zohden." In Sitzungsberichte der Kurländischen Gesellschaft für Literatur und Kunst. Mitau: J. F. Steffenhagen u. Sohn, i 888.

26 Zygmunt II August, König von Polen I 530- I 572. 
Bielski, Marcin. Kronika Polska Marcina Bielskiego. Sanok: Nakład i drukarnia Karola Pollaka, i 856.

Herzog Albrecht und Livland (I55I-I557). Regesten aus Herzogliches Briefarchiv und den Ostpreussen Folianten. Bearbeitet von Stefan Hartmann. Veröffentlichungen aus den Archiven Preußischer Kulturbesitz 57. Köln-Weimar-Wien: Böhlau, 2005.

Quellen zur Geschichte des Untergangs livländischer Selbständigkeit. Bd. 3. Herausgegeben von Carl Schirren. Reval: Franz Kluge, I 863.

Renner, Johann. Livlandische Historien I556-I56I. Bearbeitet von Peter Karstedt. Lübeck: Max Schmidt-Römhild, I 953.

Stryjkowski, Maciej. Kronika Polska, Litewska. Zmodska i wszystkiej Rusi. Bd. 2. Warszawa: Nakład Gustawa Leona Glücksberga, i 846.

\section{SECONDARY SOURCES:}

Arnold, Udo. "Hochmeister Albrecht von Brandenburg und Landmeister Gotthard Kettler. Ordensritter und Teritorialherren am Scheideweg in Preußen und Livland." In The military orders and the Reformation. Choices, state buildings and the weight of tradition, herausgegeben von Johannes A. Mol, Klaus Militzer und Helen J. Nicholson. I I-3 I. Bijdragen tot de geschiedenis der Ridderlijke Duitsche Orde, Balije van Utrecht 3. Hilversum: Verloren, 2006.

Hartmann, Stefan. "Neue Quellen zur livländischen Koadjutorfehde i 555/6." In Aus der Geschichte Alt-Livlands. Festschrift für Heinz von zur Müblen zum go. Geburtstag, herausgegeben von Bernhart Jähnig und Klaus Militzer, 275-307. Schriften der Baltischen Historischen Kommission I 2. Münster: LIT, 2004.

Klocke, Friedrich von. "Gotthard Kettler." Westfälische Lebensbilder 2 ( I 93 I ): 4 I I -438.

Kreem, Juhan. "Netzwerke um Jasper von Münster. Der Deutsche Orden während der livländischen Koadjutorfehde." Ordines Militares Colloquia Torunensia Historica. Yearbook for the Study of the Military Orders i 9 (20 I 4): 73-86.

Kreem, Juhan. "Die inneren Voraussetzungen der Säkularisation des Deutschen Ordens in Livland." Ordines Militares Colloquia Torunensia Historica. Yearbook for the Study of the Military Orders 22 (2017): 93- 108.

Lange, Thomas. Zwischen Reformation und Untergang Alt-Livlands. Der Rigaer Erzbischof Wilhelm von Brandenburg im Beziehungsgeflecht der livländischen Konföderation und ibrer Nachbarländer. Hamburg: Verlag Dr. Kovač, 20 I 4.

Maasing, Madis. "Saare-Lääne ja koadjuutorivaenus: keskaegse Liivimaa viimased kodusõjad." Ajalooline Ajakiri 2 (2010): I I 5- I 52.

Matwijów, Maciej. "Rękopiśmienne zbiory materiałów życia publicznego XVII-XVIII w. w bibliotekach i archiwach wileńskich.” In Z Badań nad Książa i Ksiegozbiorami Historycznymi, herausgegeben von Jacek Puchalski, Agnieszka Chamera-Nowak und Dorota Pietrzkiewicz, 95- ı08. Warszawa: Wydział Dziennikarstwa, Informacji i Bibliologii Uniwersytetu Warszawskiego, 20 I 7.

Mattiesen, Heinz. "Gotthard Kettler und die Entstehund des Herzogtums Kurlands." In Baltic History, herausgegeben von Mardi Valgemäe; William L. Winter und Arvids Ziedonis, 49-59. Columbus: Association for the advancement of Baltic Studies, I 974. 
Mol, Johannes A. “Traitor to Livonia? The Teutonic Orders' landmarshall Jasper von Munster and his action at the outset of the Livonian crisis, I $554-$ I 556 ." Ordines Militares Colloquia Torunensia Historica. Yearbook for the Study of the Military Orders 19 (2014): 205-240.

Morelowski, Marjan. "Zbiory dereczyńskie Sapiehów." Sprawozdania z Czynności i Posiedzeń PAU 29, Nr. г о (1 924): 6-9.

Olewnik, Jerzy. "Polsko-pruski plan inkorporacji Inflant do monarchii jagiellońskiej z lat I 552-I555 i jego pierwsze stadium realizacji." Komunikaty Mazursko-Warmińskie 4/1 46 ( I 979 ): 393-408.

Plüer, Sebastian. "Gotthard Kettler, letzter Ordenmeister in Livland und erster Herzog von Kurland - eine umstrittene Persönlichkeit in der Geschichtschreibung." In Das Herzogtum Kurland I56I-I795. Verfassung, Wirtschaft, Gesellschaft. Bd. II, herausgegeben von Erwin Oberländer, I I-53. Lüneburg: Nordostdeutsches Kulturwerk, 1993.

Popov, Vadim und Alexander Filyushkin. "Voyna koadyutorov i Pozvolskie soglasheniya I 557." Studia Slavica et Balcanica Petropolitana I (2009): I 5 I - I 85.

Radziszewski, Franciszek. Wiadomość historyczno-statystyczna o znakomitszych bibliotekach $i$ archiwach publicznych i prywatnych. Kraków: Drukarnia Wł. L. Anczyca, i 875.

Rogatschewski, Alexander. "Baltische Rechtsdenkmäler des I 3. bis i 8. Jahrhunderts im Archiv des St. Petersburger Instituts für Geschichte der Russischen Akademie der Wissenschaften." In Einheit und Vielfalt in der Rechtsgeschichte im Ostseeraum, herausgegeben von Marju Luts-Sootak, Sanita Osipova und Frank L. Schäfer, 20 I-2 I 7. Frankfurt am Main: Peter Lang $20 \mathrm{I} 2$.

Rogatschewski, Alexander. "Baltische Staats- und Rechtsgeschichte des I 3. bis i 8. Jahrhunderts in den Handschriftensammlungen von St. Petersburg." Blätter für Deutsche Landesgeschichte I 45/ 46 (2009/2010): 2 17-280.

Schiemann, Theodor. Historische Darstellungen und Archivalische Studien. Beiträge zur baltischen Geschichte. Hamburg: Gebr. Behre’s Verlag, i 886.

Seibertz, Johann. "Gotthard Kettler letzter Ordenmeister in Livland und erster Herzog von Kurland." Zeitschrift für vaterländische Geschichte und Altertumskunde Westfalen 29, Nr. 2 (I 87 I): I -92.

Weber, Dmitriy. "Pamphlet as a means of a propaganda in Baltic region in Early Modern time." Vestnik Sankt Perterburgsgovo Gosudarstvennovo Universiteta. Istoria 62/2 (2017): 29I-298.

Weber, Dmitriy. "Quellen zur Geschichte des Deutschen Ordens aus St. Petersburger Sammlungen." In Editionswissenschaftliches Kolloquium 2017. Quelleneditionen zur Geschichte des Deutschen Ordens und anderer geistlicher Institutionen, herausgegeben von Helmut Flachenecker, Krzysztof Kopiński und Janusz Tandecki, 97-ı 8. Publikationen des Deutsch-Polnischen Gesprächskreises für Quellenedition. Publikacje Niemiecko-Polskiej Grupy Dyskusyjnej do Spraw Edycji Źródeł 9. Toruń: Wydawnictwo Naukowe Uniwersytetu Mikołaja Kopernika 20 I 7. 